\title{
Bispectrum speckle interferometry observations and radiative transfer modelling of the red supergiant NML Cyg
}

\section{Multiple dust-shell structures evidencing previous superwind phases}

\author{
T. Blöcker ${ }^{1}$, Y. Balega ${ }^{2}$, K.-H. Hofmann ${ }^{1}$, and G. Weigelt ${ }^{1}$ \\ 1 Max-Planck-Institut für Radioastronomie, Auf dem Hügel 69, 53121 Bonn, Germany \\ e-mail: hofmann@mpifr-bonn.mpg.de; weigelt@mpifr-bonn.mpg.de \\ 2 Special Astrophysical Observatory, Nizhnij Arkhyz, Zelenchuk region, Karachai-Cherkesia, 35147, Russia \\ e-mail: balega@sao.ru
}

Received 16 November 2000 / Accepted 11 January 2001

\begin{abstract}
NML Cyg is a highly evolved $\mathrm{OH} / \mathrm{IR}$ supergiant, one of the most prominent infrared objects due to its strong obscuration by dust, and supposed to be among the most luminous supergiants in the galaxy. We present the first diffraction-limited $2.13 \mu \mathrm{m}$ observations of NML Cyg with 73 mas resolution. The speckle interferograms were obtained with the $6 \mathrm{~m}$ telescope at the Special Astrophysical Observatory, and the image reconstruction is based on the bispectrum speckle-interferometry method. The visibility function declines towards the diffraction limit to $\sim 0.6$. Radiative transfer calculations have been carried out to model the spectral energy distribution, given by ground-based photometry and ISO spectroscopy, and our $2.13 \mu \mathrm{m}$ visibility function. Additionally, midinfrared visibility functions at $11 \mu \mathrm{m}$ were considered. The observed dust shell properties do not appear to be in accordance with standard single-shell (uniform outflow) models but seem to require multiple components. Considering previous periods of enhanced mass-loss, various density enhancements in the dust shell were taken into account. An extensive grid of models was calculated for different locations and strenghts of such superwind regions in the dust shell. To match the observations from the optical to the sub-mm domain requires at least two superwind regions embedded in the shell. The best model includes a dust shell with a temperature of $1000 \mathrm{~K}$ at its inner radius of $6.2 R_{*}$, a close embedded superwind shell extending from $15.5 R_{*}$ to $21.7 R_{*}$ with an amplitude (factor of density enhancement) of 10 , and a far-out density enhancement at $186 R_{*}$ with an amplitude of 5 . The angular diameters of the central star and of the inner rim of the dust shell amount to 16.2 mas and 105 mas, resp. The diameter of the embedded close superwind region extends from 263 mas to 368 mas, and the inner boundary of the distant superwind region has a diameter of 3 .' 15 . In the near-infrared the dust condensation zone is limb-brightened leading to a corresponding ring-like intensity distribution. The grain sizes, $a$, were found to be in accordance with a standard distribution function, $n(a) \sim a^{-3.5}$, with $a$ ranging between $a_{\min }=0.005 \mu \mathrm{m}$ and $a_{\max }=0.15 \mu \mathrm{m}$. The bolometric flux amounts to $F_{\mathrm{bol}}=3.6310^{-9} \mathrm{Wm}^{-2}$ corresponding to a central-star luminosity of $L / L_{\odot}=1.1310^{5} \cdot(d / \mathrm{kpc})^{2}$. Within the various parts of the dust shell, $1 / r^{2}$ density distributions could be maintained differing only in their amplitude $A$. A slight improvement of the far-infrared properties can be obtained if a shallower density distribution of $\rho \sim 1 / r^{1.7}$ is considered in the distant superwind region. The present-day mass-loss rate was determined to be $\dot{M}=1.210^{-4} M_{\odot} /$ yr. The inner embedded superwind shell corresponds to a phase of enhanced mass-loss (with amplitude 10) in the immediate history of NML Cyg which began $59 \mathrm{yr}$ ago and lasted for $\sim 18 \mathrm{yr}$. Correspondingly, the outer superwind region is due to to a high mass-loss period (amplitude 5) which terminated 529 yr ago.
\end{abstract}

Key words. techniques: image processing - circumstellar matter - stars: individual: NML Cyg - stars: mass-loss - stars: supergiants - infrared: stars

\section{Introduction}

The star NML Cyg (= V1489 Cyg = IRC +40 448) belongs to the most prominent infrared objects

Send offprint requests to: T. Blöcker, e-mail: bloecker@mpifr-bonn .mpg.de of the northern hemisphere. It was discovered by Neugebauer et al. (1965) being an extremely red object in their infrared survey. This redness is due to NML Cyg's strong obscuration by circumstellar dust (e.g. Herbig \& Zappala 1970; Hyland et al. 1972). The spectral type has been determined to be M6 III (Herbig \& Zappala 1970), 
however also luminosity classes of up to Ia have been derived (e.g. Johnson 1968; Low et al. 1970).

Various distance estimates exist for NML Cyg ranging from 200 pc (Herbig \& Zappala 1970) up to 3400 pc (Rowan-Robinson \& Harris 1983). The most likely distance appears to be $\sim 2 \mathrm{kpc}$ due to the immediate proximity to the Cyg OB2 association (Morris \& Jura 1983). NML Cyg is partially surrounded by an H II region (Goss et al. 1974; Gregory \& Seaquist 1976; Habing et al. 1982) which is supposed to be the result of ionizing radiation from the Cyg OB2 association (Morris \& Jura 1983).

NML Cyg is associated with OH (Wilson et al. 1970; Masheder et al. 1974; Benson \& Mutel 1979; Bowers et al. 1983); SiO (Snyder \& Buhl 1975; Dickinson et al. 1978; Morris et al. 1979) and $\mathrm{H}_{2} \mathrm{O}$ (Schwartz \& Barrett 1970; Schwartz et al. 1974; Richards et al. 1996) maser sources. The outflow velocity for the circumstellar shell was measured to be $21 \mathrm{~km} \mathrm{~s}^{-1}$ in the $\mathrm{CO}(2-1)$ line (Knapp et al. 1982), $24 \mathrm{kms}^{-1}$ in $\mathrm{H}_{2} \mathrm{O}$ emission profiles at $22 \mathrm{GHz}$ (Engels et al. 1986), $26 \mathrm{~km} \mathrm{~s}^{-1}$ in thermal $\mathrm{SiO}$ emission at $87 \mathrm{GHz}$ (Lucas et al. 1992), and $28 \mathrm{~km} \mathrm{~s}^{-1}$ in $\mathrm{OH}$ emission profiles at $1612 \mathrm{MHz}$ (Bowers et al. 1983). Massloss rates, based on the $\mathrm{CO}$ and $\mathrm{OH}$ measurements and scaled to a distance of $1.8 \mathrm{kpc}$, are $1.510^{-4} M_{\odot} / \mathrm{yr}$ and $1.610^{-4} M_{\odot} /$ yr, resp.

Thus NML Cyg can be believed to be a highly evolved $\mathrm{OH} / \mathrm{IR}$ supergiant of very large luminosity which suffers from strong mass-loss and is highly enshrouded by dust. Morris \& Jura (1983) estimated its luminosity to be $510^{5} L_{\odot}$ at the distance of $2 \mathrm{kpc}$ (correspondig to an initial mass of $\sim 40 M_{\odot}$ ). Consequently NML Cyg appears to be among the most luminous supergiants in the Galaxy (Richards et al. 1996).

There are indications that the dust shell of NML Cyg may possibly deviate from spherical symmetry. Strong polarization was found by, e.g., Forbes (1967), Kruzewski (1971), and Dyck et al. (1971). The dust shell morphology appears to be complex. For instance, Diamond et al. (1984) found the $\mathrm{OH}$ envelope to be asymmetric, and Richards et al. (1996) concluded the existence of a bipolar outflow from interferometric observations of the $\mathrm{H}_{2} \mathrm{O}$ maser.

Previous infrared speckle interferometric observations have been reported by Sibille et al. (1979), Dyck et al. (1984), Ridgway et al. (1986), Fix \& Cobb (1988) and Dyck \& Benson (1992). Recent results from mid-infrared interferometry are given in Monnier et al. (1997), Danchi et al. (1999), and Sudol et al. (1999).

In this paper we present diffraction-limited 73 mas bispectrum speckle-interferometry observations of the dust shell of NML Cyg. Radiative transfer calculations have been performed to model the spectral energy distribution as well as visibilities at different wavelengths.

\section{Near-infrared observations and data reduction}

The NML Cyg speckle interferograms were obtained with the Russian $6 \mathrm{~m}$ telescope at the Special Astrophysical
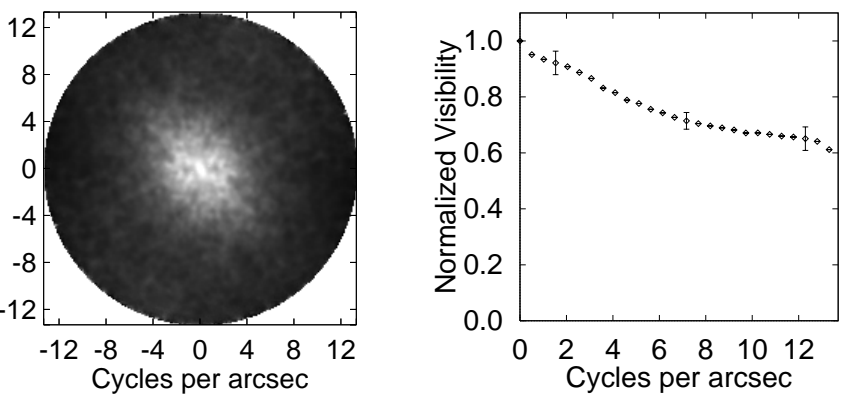

Fig. 1. Left: two-dimensional $2.13 \mu \mathrm{m}$ visibility function of NML Cyg shown up to the diffraction limit (see right panel). The bright central structure shows that the central object is surrounded by a resolved dust shell. Right: azimuthally averaged $2.13 \mu \mathrm{m}$ visibility of NML Cyg with error bars for selected frequencies

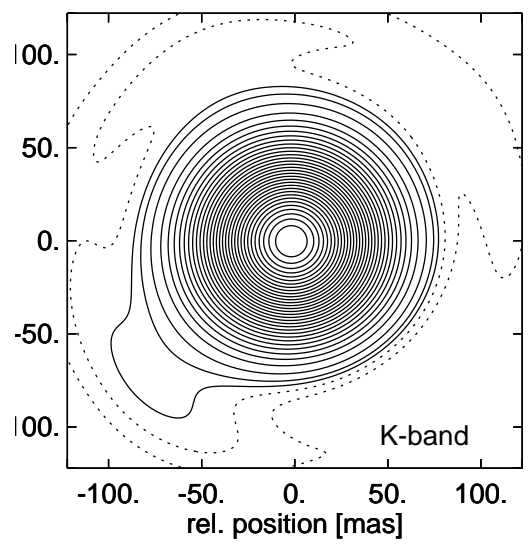

Fig. 2. Contour plot of the bispectrum speckle interferometry $2.13 \mu \mathrm{m}$-image of NML Cyg. Contour levels are plotted for $2 \%$, $3 \%, 4 \%$, and $5 \%$ of peak intensity, and from $7 \%$ to $97 \%$ of peak intensity in steps of $3 \%$. The dashed contours $(2 \%, 3 \%)$ refer to the noise floor. North is up and east is to the left

Observatory on June 13 and 14, 1998. The speckle data were recorded with our NICMOS-3 speckle camera ( $\mathrm{HgCdTe}$ array, $256^{2}$ pixels, sensitivity from 1 to $2.5 \mu \mathrm{m}$, frame rate 2 frames/s) through a continuum filter with a centre wavelength of $2.13 \mu \mathrm{m}$ and a bandwidth of $0.02 \mu \mathrm{m}$. Speckle interferograms of the unresolved star HIP 102098 were taken for the compensation of the speckle interferometry transfer function. The observational parameters were as follows: exposure time/frame $25 \mathrm{~ms}$; number of frames 5000 (2000 of NML Cyg and 3000 of HIP 102098); $2.13 \mu \mathrm{m}$ seeing $(F W H M) \sim 1$ 1. 0; field of view 7 "! $8 \times 7$ ". 8 ; pixel size 30.52 mas. A diffraction-limited image of NML Cyg with 73 mas resolution was reconstructed from the speckle interferograms using the bispectrum speckle-interferometry method (Weigelt 1977; Lohmann et al. 1983; Hofmann \& Weigelt 1986; Weigelt 1991). The bispectrum of each frame consisted of $\sim 37$ million elements. The modulus of the object Fourier transform (visibility) was determined with the speckle interferometry method (Labeyrie 1970).

Figure 1 shows the reconstructed $2.13 \mu \mathrm{m}$ visibility function of NML Cyg, whereas Fig. 2 presents a 


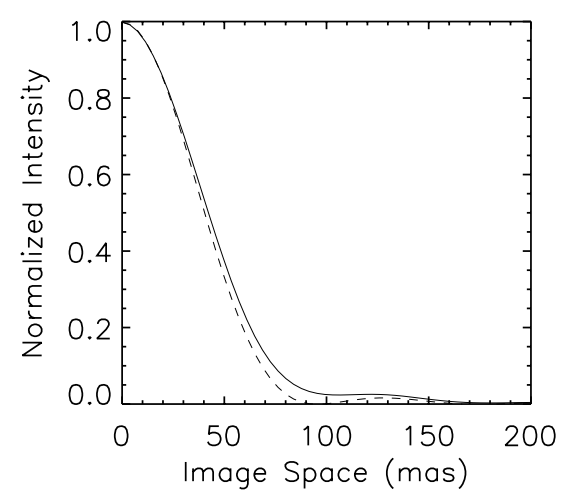

Fig. 3. Azimuthally averaged radial plots of the reconstructed diffraction-limited $2.13 \mu \mathrm{m}$-images of NML Cyg (solid line) and of the unresolved star HIP 102098 (dashed line)

contour plot of the reconstructed image. There is only little evidence for deviations from spherical symmetry. The object appears elongated at the $4 \%$ intensity level, which, however, is already close to the underlying noise floor, with a position angle of $\sim 140^{\circ}$. However, it is noteworthy that this position angle is close to the one found by $5 \mu \mathrm{m}$ interferometric measurements $\left(\sim 140^{\circ}-150^{\circ}\right.$; McCarthy 1979) and to those found for the symmetry axes of the $\mathrm{SiO}$ ( $\sim 140^{\circ}$; Boboltz \& Marvel 2000), $\mathrm{H}_{2} \mathrm{O}\left(\sim 130^{\circ}\right.$; Richards et al. 1996), and $\mathrm{OH}\left(\sim 150^{\circ}\right.$; Masheder et al. 1974) maser distributions. The Gauß fit FWHM diameter of the dust shell was determined to be 121 mas. Figure 3 displays the azimuthally averaged diffraction-limited images of NML Cyg and the unresolved star HIP 102098.

\section{Mid-infrared visibilities}

Whereas the $K$-band visibility traces the hot dust in the immediate vicinity of the star, visibility functions in the mid-infrared can give spatial information on the colder dust located further out in the circumstellar shell where the $9.7 \mu \mathrm{m}$ silicate feature forms. In the instance of NML Cyg and its heavy obscuration by dust, a considerable fraction of the observed flux is radiated at around $9.7 \mu \mathrm{m}$ and due to the thickness of the shell the silicate feature appears in absorption (see Fig. 5). Accordingly complementary $N$-band $(11.2 \mu \mathrm{m})$ interferometry can supply important constraints for the astrophysical interpretation of the object by, e.g., dust-shell modelling. Figure 4 refers to corresponding observations in this regime and shows the visibility functions at $11.2 \mu \mathrm{m}$ (Fix \& Cobb 1988), at $10.4 \mu \mathrm{m}$ and $11.4 \mu \mathrm{m}$ (Dyck \& Benson 1992), at $11.15 \mu \mathrm{m}$ (Monnier et al. 1997; Danchi et al. 1999), and at $11.5 \mu \mathrm{m}$ (Sudol et al. 1999). In the following sections we will focus on the most recent ones, i.e. the highest resolution observation of Monnier et al. (1997) and Danchi et al. (1999) with the ISI heterodyne interferometer in 1993, 1994, 1996, and 1998 and the latest low spatial frequency measurement of Sudol et al. (1999) at the $2.3 \mathrm{~m}$ Wyoming Infrared Observatory in 1997.

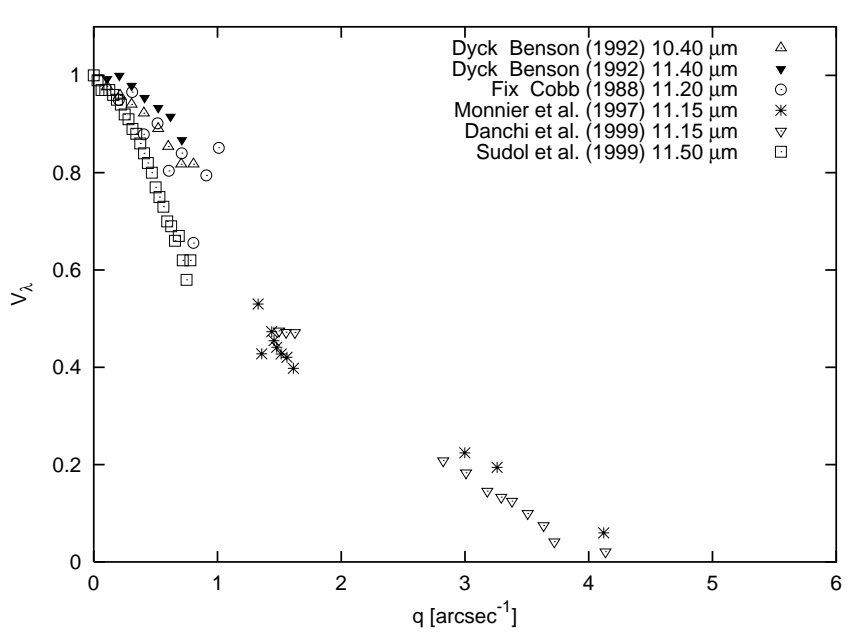

Fig. 4. Mid-infrared visibility functions of NML Cyg at $11.2 \mu \mathrm{m}$ (Fix \& Cobb 1988), at $10.4 \mu \mathrm{m}$ and $11.4 \mu \mathrm{m}$ (Dyck \& Benson 1992), at $11.15 \mu \mathrm{m}$ (Monnier et al. 1997; Danchi et al. 1999), and at $11.5 \mu \mathrm{m}$ (Sudol et al. 1999)

\section{Spectral energy distribution}

Photometric and spectrometric data in the optical and infrared for NML Cyg are given, e.g., by Johnson et al. (1965), Johnson (1967), Low et al. (1970), Hackwell (1972), Dyck et al. (1974), Strecker \& Ney (1974) and Merrill \& Stein (1976). NML Cyg shows small long-term variations in its spectral energy distribution (Harvey et al. 1974; Strecker 1975) with a period of $\sim 1000$ days. Recently, Monnier et al. (1997) presented long-term $10.2 \mu \mathrm{m}$ photometry covering a time basis of more than $12 \mathrm{yr}$. They determined an amplitude of $\sim 0.5 \mathrm{mag}$ and a mean period of $\sim 940$ days. Thus NML Cyg can be considered as a semiregular variable. Monnier et al. $(1997,1998)$ investigated the temporal variation of the mid-infrared spectra by means of $8-13 \mu \mathrm{m}$ spectrophotometry from 1991 to 1995 finding an almost constant spectral shape in this wavelength regime. Submillimeter $(400 \mu \mathrm{m})$ and millimeter $(1.3 \mathrm{~mm})$ observations are given by Sopka et al. (1985) and Walmsley et al. (1991), resp. Besides these ground-based observations NML Cyg has also been observed by IRAS (Infrared Astronomical Satellite) in 1973 and by ISO (Infrared Space Observatory) in 1996.

NML Cyg is highly reddened by the interstellar medium and the circumstellar shell. Low et al. (1970) estimated a total extinction of $A_{V}^{\text {total }} \approx 9.5$ for NML Cyg. Based on radio continuum observations Gregory \& Seaquist (1976) obtained an interstellar extinction of $A_{V} \approx 4 .{ }^{\mathrm{m}} 6$ whereas Lee $(1970)$ estimated $E(B-V)=1{ }^{\mathrm{m}} 2$ for the interstellar contribution. We will use the latter value of $E(B-V)=1 \mathrm{~m} 2$. This interstellar reddening was taken into account by adopting the method of Savage \& Mathis (1979) with $A_{V}=3.1 E(B-V)$.

The spectral energy distrubion (SED) of NML Cyg is shown in Fig. 5 and exhibits a $9.7 \mu \mathrm{m}$ silicate profile in absorption. It includes the (dereddened) data of Johnson et al. (1965), Dyck et al. (1974) and Merrill \& Stein (1976) 

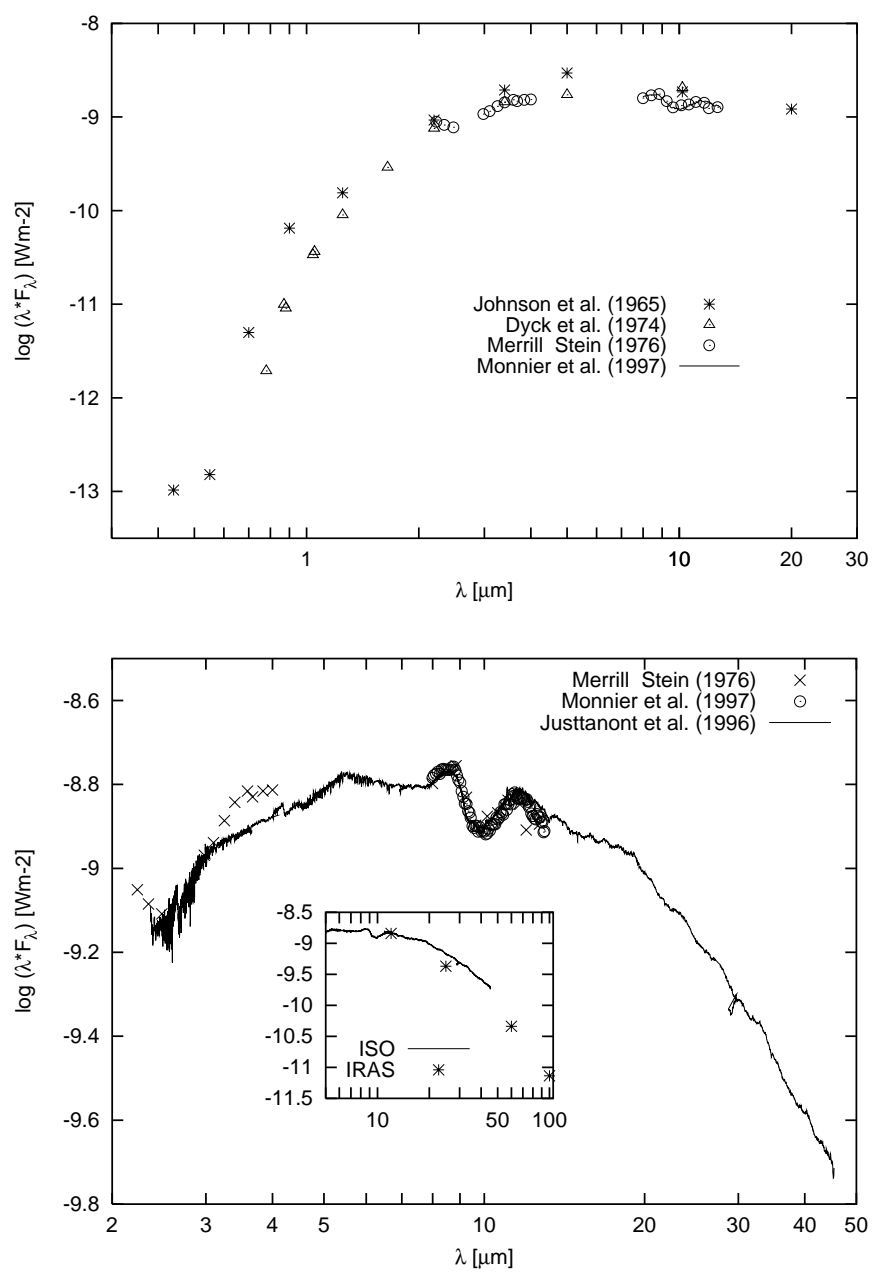

Fig. 5. Spectral energy distribution of NML Cyg (corrected for interstellar extinction of $\left.A_{V}=3.7\right)$. Top: photometry of Johnson et al. (1965), Dyck et al. (1974) and Merrill \& Stein (1976) as well as the UKIRT spectrophotometry of Monnier et al. (1997). The Merrill \& Stein (1976) data was scaled to match the UKIRT photometry. Bottom: ISO-SWS spectrum of Justtanont et al. (1996), UKIRT spectrophotometry of Monnier et al. (1997), and IRAS photometry. The ISO data was adjusted to match the ground-based photometry (see also Justtanont et al. 1996)

as well as the UKIRT spectrophotometry of Monnier et al. (1997). Note that the Merrill \& Stein (1976) data has been scaled to match the UKIRT spectrophotometry as in Monnier et al. (1997). The spectral shapes of both data sets agree well. The lower panel of Fig. 5 shows the ISOSWS spectroscopy (Justtanont et al. 1996) in comparison with the UKIRT data and the IRAS photometry. The ISO data was adjusted to match the ground-based photometry (see also Justtanont et al. 1996). In the long-wavelength region, the IRAS fluxes seem to be somewhat smaller than those provided by ISO.

\section{Dust shell models}

In order to model both the observed SED and the $2.13 \mu \mathrm{m}$ and $11.15 \mu \mathrm{m}$ visibilities, radiative transfer calculations for the dust shell were conducted. For the robust and non-ambiguous construction of dust shell models it is essential to take diverse and independent observational constraints into account. Apart from matching the spectral energy distribution, the consideration of spatially resolved information plays a crucial role for obtaining a reliable model (see e.g. Blöcker et al. 1999).

\subsection{The radiative transfer code}

We used the code DUSTY developed by Ivezić et al. (1997), which solves the radiative transfer problem in spherical symmetry. It considers absorption, emission and scattering and utilizes the self-similarity and scaling behaviour of IR emission from radiatively heated dust (Ivezić \& Elitzur 1997). To solve the radiative transfer problem the spectral shape of the central source's radiation has to be specified and various properties of the surrounding dust shell are required, viz. (i) the chemical composition and grain size distribution; (ii) the dust temperature at the inner boundary; (iii) the relative thickness, i.e. the ratio of outer to inner shell radius; (iv) the density distribution; and (v) the total optical depth at a given reference wavelength. The code has been expanded for the calculation of synthetic visibilities as described by Gauger et al. (1999).

\subsection{Single-shell models}

Dust shell models for NML Cyg were reported in RowanRobinson \& Harris (1983), Rigdway et al. (1986), and Monnier et al. (1997). The first two studies used uniform outflow models, i.e. approximated the density distribution of the dust shell by $\rho \sim r^{-2}$. Monnier et al. (1997) found that uniform outflow models appear not to be consistent with their near- and mid-infrared observations and proposed a double-shell structure for the dust envelope. It should be kept in mind, however, that the older calculations are partly based on different input quantities, as optical constants, grain sizes, or shape of the central source radiation.

We calculated various uniform outflow models considering the following parameters within the radiative transfer calculations: SED and visibilities were modelled for effective temperatures of $T_{\text {eff }}=2000$ to $3000 \mathrm{~K}$ and black bodies as central sources of radiation. We used the silicates of Ossenkopf et al. (1992) which were found to be well suited to fit the silicate feature by Monnier et al. (1997). Some test calculations were performed with the optical constants of of Draine \& Lee (1984) and David \& Pegourie (1995) as well. For the grain-size distribution we considered grain size distributions according to Mathis et al. (1977, hereafter MRN), i.e. $n(a) \sim a^{-3.5}$, with $0.005 \mu \mathrm{m} \leq a \leq(0.13$ to 0.45$) \mu \mathrm{m}$ as well as singlesized grains with $a=0.05$ to $0.3 \mu \mathrm{m}$. The shell thickness $Y_{\text {out }}=r_{\text {out }} / r_{1}$ amounted to $10^{3}$ to $10^{4}$ with $r_{\text {out }}$ and $r_{1}$ being the outer and inner radius of the shell, respectively. With these quantities specified, the remaining fit 

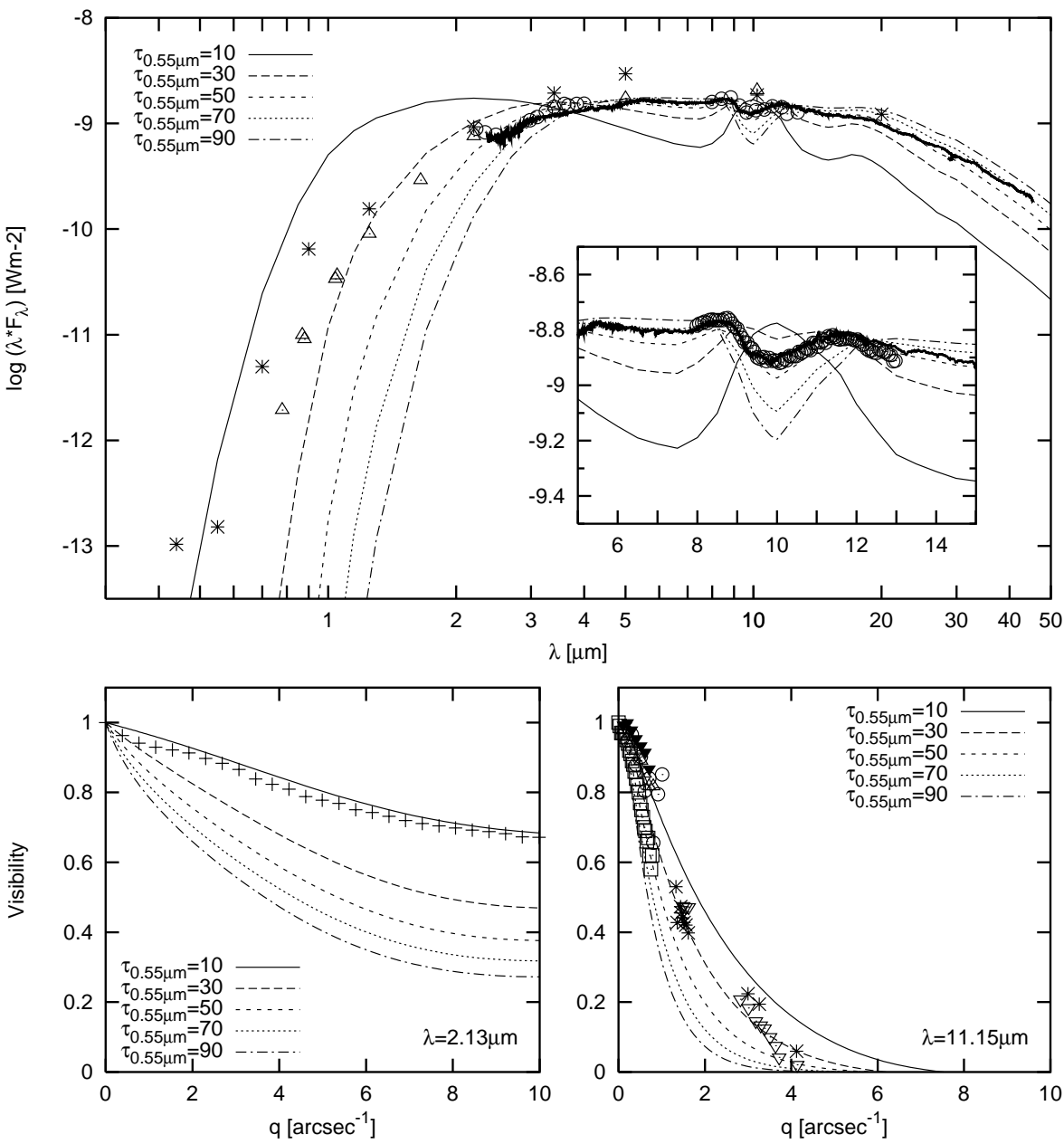

Fig. 6. Optical depth dependence of model SED (top) and model visibilities at $2.13 \mu \mathrm{m}$ (bottom left) and $11.15 \mu \mathrm{m}$ (bottom right) for $T_{\text {eff }}=2500 \mathrm{~K}$ and $T_{1}=1000 \mathrm{~K}$. The optical depth $\tau(0.55 \mu \mathrm{m})$ ranges between 10 and 90 . The calculations are based on a black body, Ossenkopf et al. (1992) silicates, a Mathis et al. (1977) grain size distribution with $a_{\max }=0.25 \mu \mathrm{m}$, and a $1 / r^{2}$ density distribution. Such a standard model does not appear applicable to NML Cyg since different optical depths are required to match the various observations. The symbols refer to the observations (see text, Figs. 1, 4 and 5) corrected for interstellar extinction of $A_{V}=3.7$ :

SED: *: Johnson et al. (1965), $\triangle$ : Dyck et al. (1974), ॰: Merril \& Stein (1976), thick line: Justtanont et al. (1996) [ISO data]. The inlet shows the ISO data of Justtanont et al. (1996) (thick line) and the spectrophotometry of Monnier et al. (1997) (circles). Visibilities: +: $2.13 \mu \mathrm{m}$ (present paper);

$\triangle: 10.4 \mu \mathrm{m}$ (Dyck \& Benson 1992), $\mathbf{v}: 11.4 \mu \mathrm{m}$ (Dyck \& Benson 1994), o: $11.2 \mu \mathrm{m}$ (Fix \& Cobb 1988), *: 11.15 $\mu \mathrm{m}$ (Monnier et al. 1997), $\nabla: 11.15 \mu \mathrm{m}$ (Danchi et al. 1999), $\square: 11.5 \mu \mathrm{m}$ (Sudol et al. 1999).

parameters are the dust temperature at the inner boundary, $T_{1}$, which, in turn, determines the radius of the shell's inner boundary, $r_{1}$, and the optical depth, $\tau$, at a given reference wavelength, $\lambda_{\text {ref }}$. We refer to $\lambda_{\text {ref }}=0.55 \mu \mathrm{m}$. Models were calculated for dust temperatures between 400 and $1500 \mathrm{~K}$ and optical depths between 10 and 100 .

The neccessity to consider various observational constraints in order to obtain dust-shell properties by radiative transfer calculations is demonstrated in Fig. 6. It shows that the SED and the near-infrared and midinfrared visibilities cannot be fitted simultaneously by a given set of parameters. Though each single observation can be matched reasonably well, different values for the optical depth are required. For example, the $K$-band visibility requires $\tau(0.55 \mu \mathrm{m})=10$, the $N$-band visibility $\tau(0.55 \mu \mathrm{m})=30$, and the silicate feature $\tau(0.55 \mu \mathrm{m})=50$ for the chosen dust-shell properties. High optical depths $(\tau(0.55 \mu \mathrm{m}) \gtrsim 30)$ lead to a flux deficit in the optical.

This example refers to a given dust temperature of $T_{1}=1000 \mathrm{~K}$ at the inner boundary and an MRN grainsize distribution $n(a)$ with $a_{\max }=0.25 \mu \mathrm{m}$. Additionally the models rely on the assumption of a standard density distribution $\rho(r) \sim 1 / r^{2}$ whose validity has been questioned by Monnier et al. (1997). Before abandoning the uniform outflow model, however, one has to consider the effects of changes of the former two dust-shell properties, $T_{1}$ and $n(a)$.

The $K$-visibility is very sensitive against scattering and, thus, depends strongly on the assumed grain sizes. Increasing the (maximum) grain size has a similar effect 

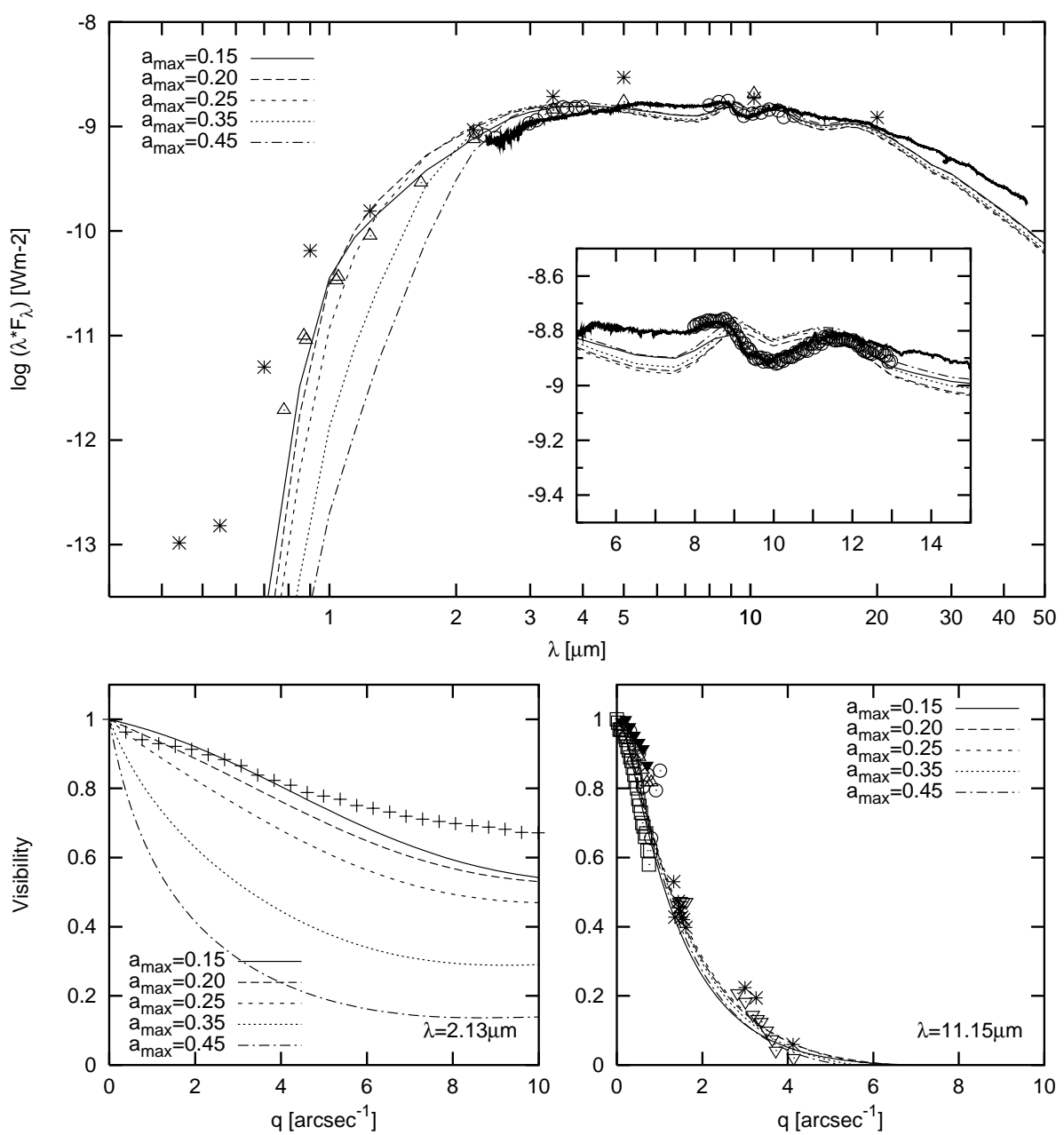

Fig. 7. Maximum grain-size dependence of model SED (top) and model visibilities at $2.13 \mu \mathrm{m}$ (bottom left) and $11.15 \mu \mathrm{m}$ (bottom right) for $T_{\text {eff }}=2500 \mathrm{~K}, T_{1}=1000 \mathrm{~K}, \tau(0.55 \mu \mathrm{m})=30$. The maximum grain size $a_{\text {max }}$ ranges between $0.15 \mu \mathrm{m}$ and $0.45 \mu \mathrm{m}$. The calculations are based on a black body, Ossenkopf et al. (1992) silicates, a Mathis et al. (1977) grain size distribution, $n(a) \propto a^{-3.5}$, and a $1 / r^{2}$ density distribution. The symbols refer to the observations (cf. Figs. 1, 4 and 5 ) corrected for interstellar extinction of $A_{V}=3.7$ and are the same as those in Fig. 6

as increasing the optical depth and results into a stronger decline of the $K$-visibility (cf. e.g. Blöcker et al. 1999). This holds both for single-sized grains and grain-size distributions. If one increases the maximum grain-size of an MRN-like distribution most particles are still small due to the steep decline of the distribution function towards larger grain sizes. However, due to the very strong dependence of the absorption and scattering properties on the grain sizes, these "few" larger particles determine the $K$ visibility equally as much as the bulk of the smaller grains (Krüger \& Sedlmayr 1997; Winters et al. 1997). On the other hand, at longer wavelengths the changes induced by a larger maximum grain size are predominantly of minor nature. For instance, the SED silicate feature and the mid-infrared visibility are only mildly affected. This fact allows, in principle, to fix the optical depth by matching the silicate feature and mid-infrared visibilities (if possible) and to fix independently the grain-size distribution by the near-infrared data. The impact of maximum grain sizes of MRN-like distribution on the various observational quantities is illustrated in Fig. 7. These calculations show that the low-frequency part of the $K$-band visibility may be fitted for smaller optical depths if $a_{\max }$ is sufficiently small. However, the silicate feature and the SED's longwavelength tail are then only poorly matched. The most favorable maximum grain size within an MRN-like distribution was found to be close to $a_{\max }=0.15 \mu \mathrm{m}$.

To complete the discussion on grain sizes it has to be considered how an MRN-like distribution compares with single-sized grains and which choice is better suited for the instance of NML Cyg. Whereas the $K$-visibility behaves similarly in both cases, i.e. strongly declines with for increasing grain sizes, SED and mid-infrared visibilities are more affected by variations of single-sized grains than by those of a distribution. The interplay between optical depth and grain size is now much stronger. In summary, we found that single-sized grains appear to give worse overall fits compared to grain-size distributions.

Finally, we want to address the question of the dust temperature at the inner shell boundary. The hotter the 
dust at the inner boundary of the shell, the more flux is radiated in the near-infrared and the less dominant is the long-wavelength tail of the SED. Concomitantly, the visibilities decline at low spatial frequencies the steeper, the hotter the inner dust-shell rim. Inspection of the model grid shows that the dust temperature at the inner boundary should be close to $1000 \mathrm{~K}$. Somewhat higher values, e.g. $1200 \mathrm{~K}$, are possible as well.

All parameter variations discussed so far (of optical depths, dust temperatures, grain sizes etc.) failed to give a consistent overall fit, thus the only basic parameter left to vary is the density distribution (assuming spherical symmetry). Consequently, it can be concluded that the assumption of a uniform outflow model does not hold for NML Cyg and that, for instance, steeper density distributions or multiple shells have to be considered. A similar conclusion was drawn by Monnier et al. (1997) who introduced two shells with exponentially decreasing density distributions in order to match the observations.

\subsection{Multiple dust-shell components}

The evolution of massive supergiants can be accompanied by stages of episodic mass-loss events as superwind phases or even mass-loss outbursts, as for example, during the Red Supergiant Branch or the Luminous Blue Variable stage (see, e.g., Vanbeveren et al. 1994; Heger et al. 1997; de Jager \& Nieuwenhuijzen 1997; Langer et al. 1998). During transitory phases of heavy mass-loss the density in the dust shell is increased. This leads to regions in the dust shell with density enhancements over the normal $1 / r^{2}$ distribution. The radial density distribution may also change within such superwind shells. For more details, see Suh \& Jones (1997). For example, such superwind models have successfully been applied to the hypergiant IRC +10420 that is believed to have already moved off the RSG currently evolving into the Wolf-Rayet stage (see Blöcker et al. 1999).

Concerning the introduction of superwind models different degrees of complexity can be considered: (i) single jumps with enhancement factors, or amplitudes, $A$ at radii $Y_{n 1}=r_{n 1} / r_{1}$; (ii) a single superwind shell with amplitude $A$ ranging from $Y_{n 1}$ to $Y_{n 2}$; (iii) various shells; and (iv) jumps or shells as in (i)-(iii) but with density distributions different from the $1 / r^{2}$ uniform outflow model. Examples of the superwind models (i)-(iii) are illustrated in Fig. 8.

In the following sections such superwind models will be discussed in more detail. In each case the suitability of other dust-shell parameters was carefully checked. We found a optical depth of $\tau(0.55 \mu \mathrm{m})=30$ to fit the silicate feature. Within an MRN-like grain-size distribution a maximum grain-size of $a_{\max }=0.15 \mu \mathrm{m}$ was chosen in order to match the overall pattern of the SED and the lowfrequency curvature of the $K$-band visibility. We stayed with the optical constants of Ossenkopf et al. (1992) due to their excellent match of the silicate feature's shape. The dust-temperature at the inner dust-shell boundary

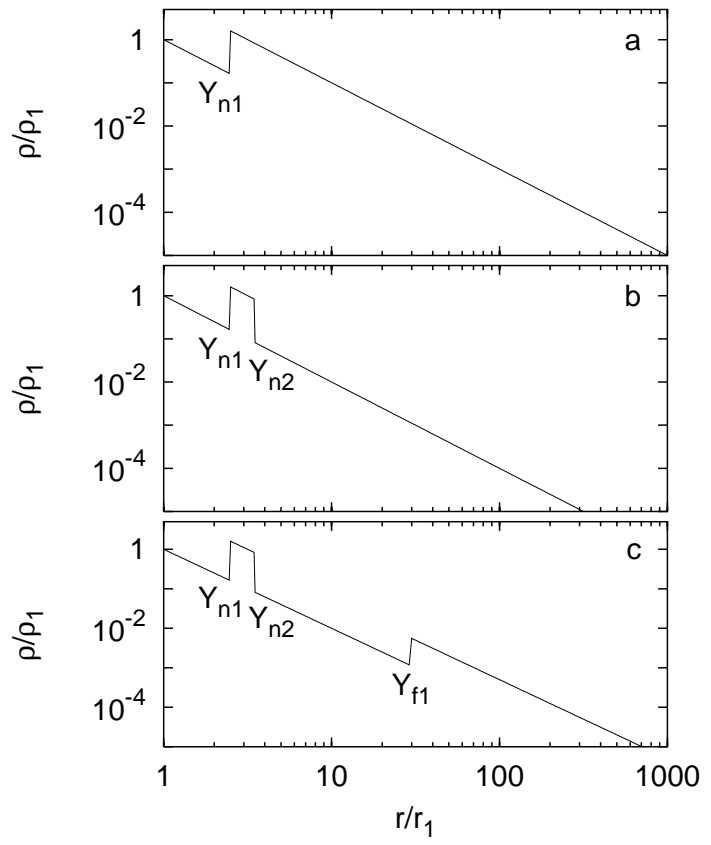

Fig. 8. Relative density distribution for different superwind models with $\rho \sim 1 / r^{2}$. The upper panel a) shows a density enhancement at $Y_{n 1}=r_{n 1} / r_{1}=2.5$, i.e. nearby the inner dustshell rim, with an amplitude of $A=10$. The middle panel $\mathbf{b}$ ) refers to a superwind shell ranging from $Y_{n 1}=2.5$ to $Y_{n 2}=3.5$ with $A=10$, and the bottom panel c) shows this superwind shell and an additional far-out density enhancement at $Y_{f 1}=$ 30 with $A=5$

amounted to $1000 \mathrm{~K}$, the effective temperature of the central star to $2500 \mathrm{~K}$.

\subsubsection{Two-component shells}

The most simple model of a previous epoch of enhanced mass-loss is that of a single density enhancement as shown in the upper panel of Fig. 8. Calculations were conducted for relative distances $Y_{n 1}$ between 1.5 and 6.5 and amplitudes $A$ ranging from 10 to 20 . The best model found is that with $Y_{n 1}=2.5$ and $A=10$ which fits the SED from the optical to the infrared and matches the $K$-band visibility reasonably well. The inner boundary of the dust shell amounts to $r_{1}=5.8 R_{*}\left(T_{1}=1000 \mathrm{~K}\right)$ corresponding to an angular diameter of 95 mas. At $Y_{n 1}=2.5$ the dust temperature has dropped to $621 \mathrm{~K}$. More distant density jumps lead to worse fits of the silicate feature and the far-infrared regime, less distant ones are not in agreement with the $K$-band visibility (for given amplitude). However, the mid-infrared visibility was only poorly matched by all of these models giving too extended shells at this wavelength. This, in turn, indicates that the cold dust-shell regions which determine the mid-infrared visibility have too high densities within this model. 


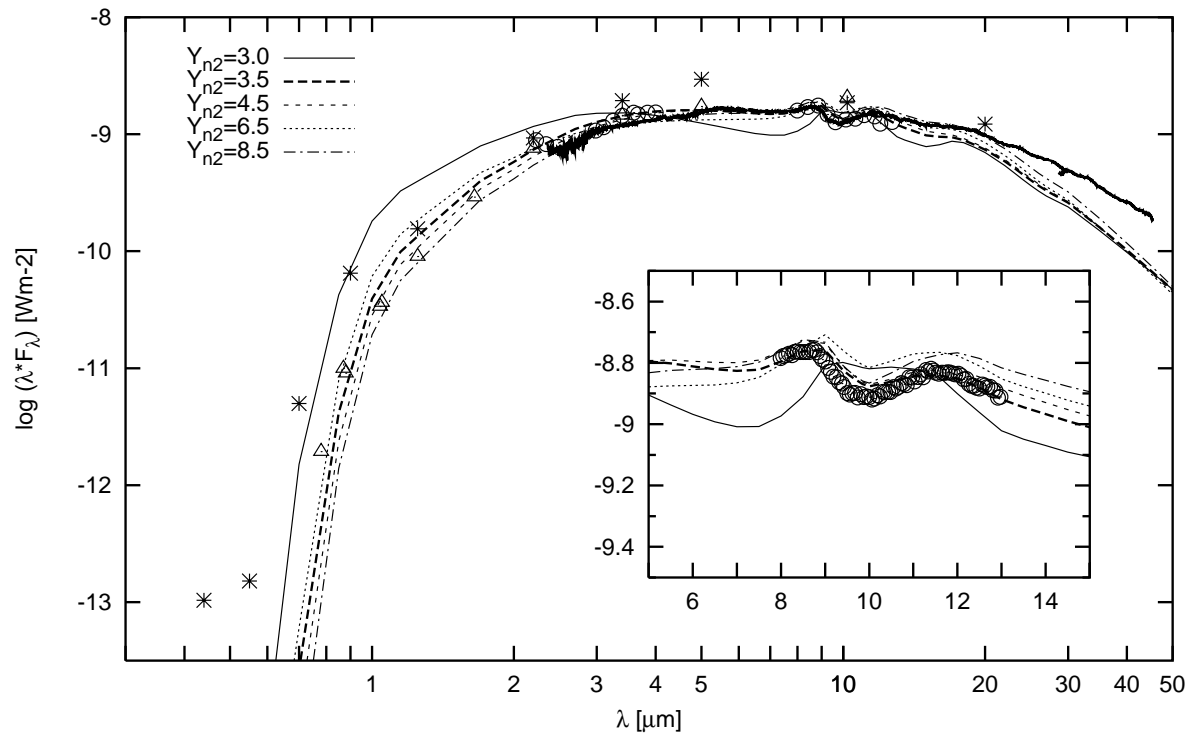

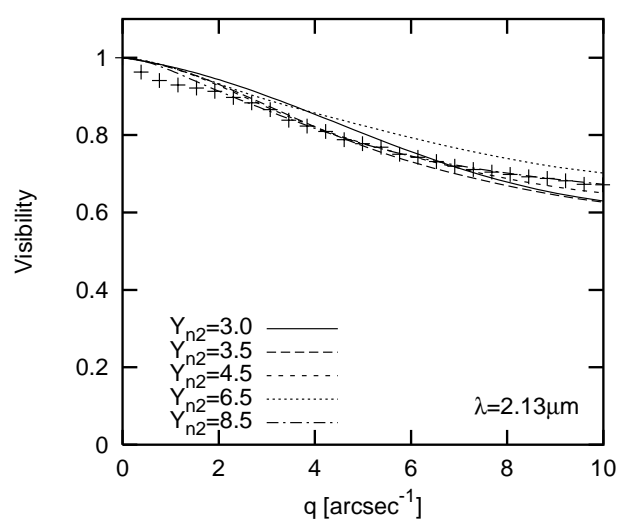

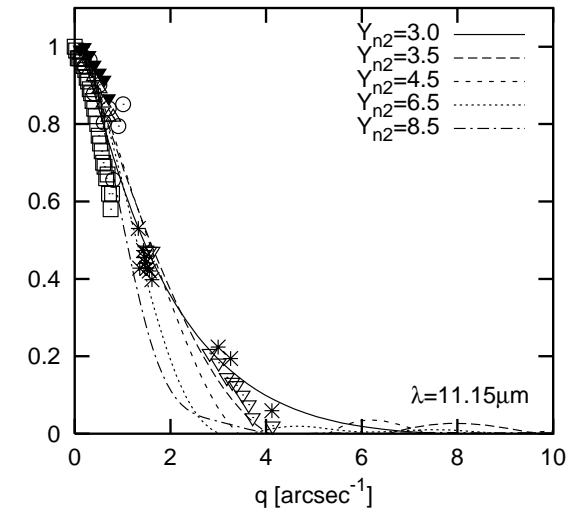

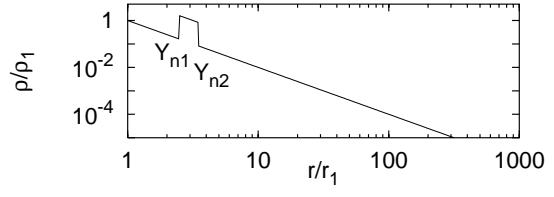

Fig. 9. Model SED (top, left) and model visibilities at $2.13 \mu \mathrm{m}$ (bottom left) and $11.15 \mu \mathrm{m}$ (bottom right) for $T_{\text {eff }}=2500 \mathrm{~K}, \tau(0.55 \mu \mathrm{m})=30, T_{1}=$ $1000 \mathrm{~K}$, and different embedded superwind shells with amplitude $A=10$ extending from $Y_{n 1}=2.5$ to $Y_{n 2}=3.0$ 8.5. The density distribution is exemplified for $Y_{n 1}=2.5$ and $Y_{n 2}=3.5$ in the above panel (top, right) (cf. Fig. 8). The calculations are based on a black body, Ossenkopf et al. (1992) silicates, and a Mathis et al. (1977) grain size distribution with $a_{\max }=0.15 \mu \mathrm{m}$. The symbols refer to the observations (cf. Figs. 1, 4 and 5) corrected for interstellar extinction of $A_{V}=3.7$ and are the same as those in Fig. 6

\subsubsection{Embedded superwind shells}

Since the $K$-band visibility traces the hot dust, it is sensitive to the place and strength of the density jump but reacts only moderately to the extension of the density enhancement. Consequently, the above model can be improved by introducing an embedded high-density or superwind shell of given extension (see Fig. 8, middle panel) instead of a single jump. Since the inner boundary and the amplitude of such a shell is strongly constrained by the $K$ band visibility, the inner boundary was fixed to $Y_{n 1}=2.5$ and the amplitude to $A=10$ within the corresponding model grid. Additional test calculations with other values for $Y_{n 1}$ and $A$ were performed as well. The outer boundary $Y_{n 2}$ was varied between 3.0 and 10.5. SED and visibility models for different values of $Y_{n 2}$ are shown in Fig. 9. It turned out that only a close and comparatively thin shell ranging from $Y_{n 1}=2.5$ to $Y_{n 1}=3.5$ appears to be in agreement with both the visibility data at different wavelengths and the SED from the optical to the mid-infrared. Now, the inner boundary of the dust shell is located somewhat more outwards, viz. at $R_{1}=6.2 R_{*}$ corresponding to an angular diameter of 101 mas. The superwind shell diameter extends from 252 to 353 mas, the corresponding dust temperature at the inner and outer boundary amount to $587 \mathrm{~K}$ to $411 \mathrm{~K}$, resp. However, as obvious from Fig. 9 the model appears to be still incomplete due to a lack of sufficiently high fluxes at longer wavelengths $(\lambda \gtrsim 20 \mu \mathrm{m})$.

\subsubsection{Two superwind shells}

The above model of a thin density-enhanced region close to the inner boundary of the dust shell gives a reasonable fit to the observed properties from the optical to the midinfrared. However, due to the small extension of the embedded superwind shell, the densities in the outer regions of the dust shell appear to be too small to provide sufficiently high far-infrared fluxes. This may indicate either the existence of a second, far-out density enhancement or a shallower radial density dependence (e.g. $\rho \sim 1 / r^{1.5}$ ). In the case of a second episodic mass-loss phase, the corresponding density enhancement should be at moderate distances, close enough to raise the fluxes at $\lambda>20 \mu \mathrm{m}$ but distant enough in order to preserve the properties of the radiative transfer models at shorter wavelengths.

Grids were calculated for such an additional far-out superwind shell with an inner boundary located between $Y_{f 1}=7$ to 50 and amplitudes $A$ ranging between 2 and 50. The best model found was that with $Y_{f 1}=30$ and a moderate amplitude of $A=5$. For simplicity we considered here only density jumps as shown in Fig. 8. Note that though the existence of such a distant density 
enhancement appears to be well constrained by the observations within the current model, the corresponding properties of the distant shell are less constrained. For instance, different combinations of location, amplitude and density slope can lead to models of comparable quality. Hovever, shells at significantly smaller or larger distances can clearly be excluded.

Since the introduction of a far-out density enhancement hardly affects the inner regions, the inner boundary of the dust shell keeps at 6.2 stellar radii corresponding to an angular diameter of 105 mas. The angular diameter increased somewhat compared to the case without an outer shell due to the (slight) increase of the bolometric flux. The diameter of the embedded close superwind shell extends from 263 mas to 368 mas, and the inner boundary of the distant shell has a diameter of $3{ }^{\prime \prime} 15$. The dust temperature within the embedded superwind shell ranges from $584 \mathrm{~K}$ to $430 \mathrm{~K}$ and within the distant shell from $149 \mathrm{~K}$ to $40 \mathrm{~K}$.

Figure 11 gives the fractional flux contributions of the emerging stellar radiation, of the scattered radiation and of the thermal dust emission as a function of wavelength for this model. At $2.2 \mu \mathrm{m}$, the flux is mainly determined by direct stellar light $(67 \%)$. Scattered radiation and dust emission contribute $6 \%$ and $27 \%$, resp. Thus, roughly $80 \%$ of the circumstellar shell's $K$-band radiation is due to thermal dust emission. At wavelengths larger than $10 \mu \mathrm{m}$ dust emission completely dominates. The bolometric flux amounts to $F_{\mathrm{bol}}=3.6310^{-9} \mathrm{Wm}^{-2}$. Accordingly, the central star has a luminosity of $L / L_{\odot}=1.1310^{5} \cdot(d / \mathrm{kpc})^{2}$ and an angular diameter of $\Theta_{*}=1.7410^{9} \sqrt{F_{\mathrm{bol}} / T_{\mathrm{eff}}^{4}} \sim$ 16.2 mas.

Figure 12 shows the normalized intensity distribution at $2.13 \mu \mathrm{m}$ (upper panel) and $11.15 \mu \mathrm{m}$ (lower panel) as function of angular distance. The barely resolved central peak corresponds to the central star (angular diameter: 16.2 mas). At $2.13 \mu \mathrm{m}$ the inner boundary of the dust shell (radius: 52.5 mas) is limb-brightened resulting in a ring-like intensity distribution. The optical depth $(\tau(2.13 \mu \mathrm{m})=1.5)$ is still moderate leading to a noticable effect of a limb-brightened dust condensation zone (cf. Ivezic \& Elitzur 1996; Blöcker et al. 1999). In the $N$ band $(\tau(11.5 \mu \mathrm{m})=2.2)$ this is hardly visible. The embedded superwind shell leads to an increase of the intensity distribution's waist at angular radii between 131.5 mas and 184 mas. Finally, the far-out shell provides broad lowintensity wings at angular radii larger than $\sim 1{ }^{\prime \prime} .5$.

Asuming an outflow velocity of $25 \mathrm{~km} \mathrm{~s}^{-1}$ and a distance of $1.8 \mathrm{kpc}$, the kinematical ages of these shells can be calculated. The inner embedded shell corresponds to a phase of enhanced mass-loss lasting for $\sim 18 \mathrm{yr}$ in the immediate history of NML Cyg. It began $59.2 \mathrm{yr}$ ago and ceased 41.5 yr ago. During this time span the mass-loss rate increased by a factor of 10 . Correspondingly, the outer shell, i.e. the density jump at $Y_{f 1}=30$, is due to to a high mass-loss period which terminated $529.3 \mathrm{yr}$ ago. Throughout this phase in the past matter was expelled with a fivefold increased rate. With a dust-to-gas ratio of 0.005 and a specific dust density of $3 \mathrm{~g} \mathrm{~cm}^{-3}$ the model gives a present-day mass-loss rate of $\dot{M}=$ $1.210^{-4} M_{\odot} / \mathrm{yr}$ which is in line with the $\mathrm{CO}$ and $\mathrm{OH}$ observations (see Sect. 1).

\subsubsection{Other density slopes}

In the above sections the uniform-outflow model was abandoned due to its mismatch of the observed properties. The introduction of two superwind shells, or density enhancements of certain amplitude $A$, leads to a model which fits the observations from the optical to the infrared. However, so far it was assumed that $\rho \sim 1 / r^{2}$ holds within each dust-shell region. Assuming the outflow velocity kept constant any shallower (steeper) density slope than $\rho \sim 1 / r^{2}$ means that the mass-loss rate has decreased (increased) with time in the respective dust-shell regions.

The double-shell superwind models still seem to predict a somewhat larger spectral index, i.e. faster flux decrease, than the ISO observations indicate for $\lambda \gtrsim 20$ (Fig. 10), and than $400 \mu \mathrm{m}$ and $1.3 \mathrm{~mm}$ data of Sopka et al. (1985) and Walmsley et al. (1991), resp., show. Additional models with shallower density distributions in the outer shell were calculated to improve the model. A somewhat shallower distribution of $\rho \sim 1 / r^{1.7}$ in the far-out regions $(Y \geq 30)$ of the dust shell appears to be slightly better suited than the standard model with $\rho \sim 1 / r^{2}$ and matches also the $400 \mu \mathrm{m}$ observations. The $1.3 \mathrm{~mm}$ data, however, cannot be matched by any of the models. It has already been noted by Sopka et al. (1985) and Walmsley et al. (1991) that the fluxes at $400 \mu \mathrm{m}$ and $1.3 \mathrm{~mm}$ are in excess of standard models. It has been argued that flat density distributions (as introduced above) or an increased emissivity of the silicates may be responsible for this excess.

If one considers different density slopes in the outer shell to improve the model, the question arises if this should not be considered for the whole dust shell model in general. For instance, Monnier et al. (1999) found two shells with exponentially decreasing density distributions appropriate to match the observations. In order to prove (or disprove) the applicability of the assumed $1 / r^{2}$ distribution in the inner regions of the shell $(Y \leq 30)$, we re-calculated the main body of the grid presented so far including the superwind shells for density distribution ranging from $\rho \sim 1 / r$ to $\rho \sim 1 / r^{7}$ and combinations of it. We found the same best model as presented above to match the observations from the optical to the sub-mm domain, i.e. a (close-to) $1 / r^{2}$ overall density distribution with a thin superwind shell $(Y=2.5$ to 3.5$)$ near to the inner dust-shell rim and a far-out density enhanced region $(Y \gtrsim 30)$. Shallower distributions in the inner region can be excluded, for instance due to the mismatch of the mid-infrared visibility. Steeper distributions partly match the observations but were found to be unable to provide an overall fit. If one considers only the embedded superwind shell, models can be constructed which show, 

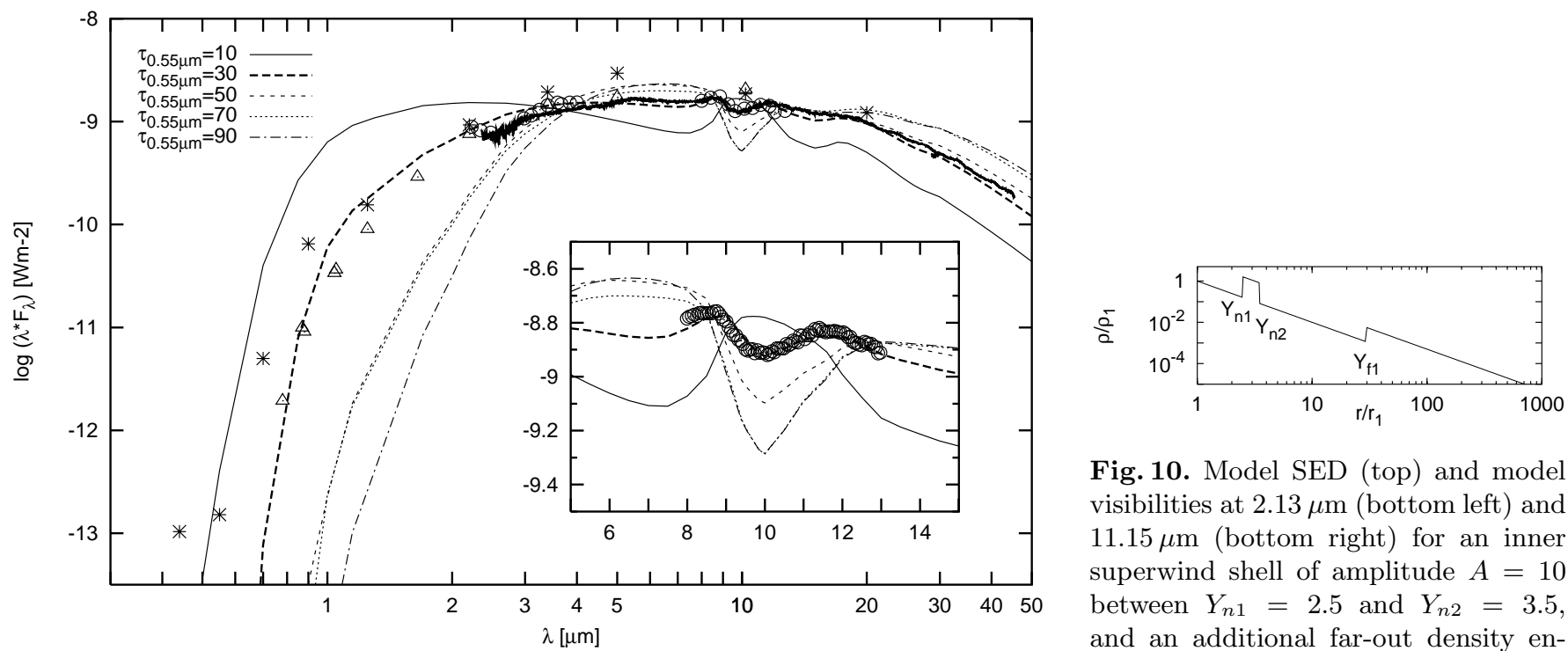

Fig. 10. Model SED (top) and model visibilities at $2.13 \mu \mathrm{m}$ (bottom left) and $11.15 \mu \mathrm{m}$ (bottom right) for an inner superwind shell of amplitude $A=10$ between $Y_{n 1}=2.5$ and $Y_{n 2}=3.5$, and an additional far-out density en-
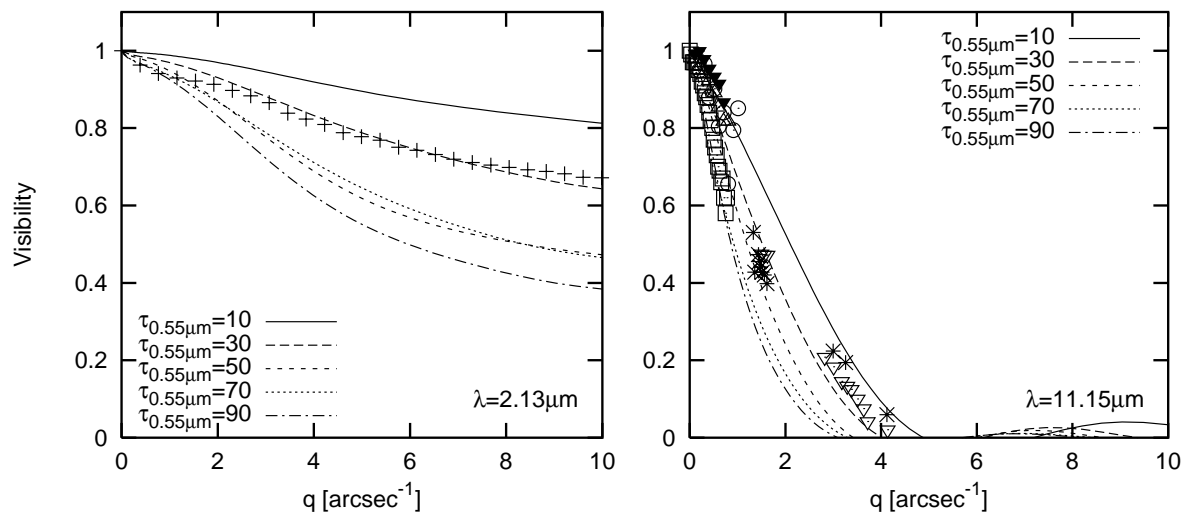
hancement at $Y_{f 1}=30$ with $A=5$. The density distribution is displayed in the above panel (top, right) (cf. Fig. 8). The models refer to $T_{\text {eff }}=$ $2500 \mathrm{~K}, T_{1}=1000 \mathrm{~K}$ and optical depths $\tau(0.55 \mu \mathrm{m})$ ranging between 10 and 90. The calculations are based on a black body, Ossenkopf et al. (1992) silicates, and a Mathis et al. (1977) grain size distribution with $a_{\max }=0.15 \mu \mathrm{m}$. The symbols refer to the observations (cf. Figs. 1, 4 and 5) corrected for interstellar extinction of $A_{V}=3.7$ and are the same as those in Fig. 6

in principle, similar properties as the one presented above but have a much steeper density distribution within the embedded superwind shell, i.e. as steep as $1 / r^{5-7}$. Then the superwind shell has to be somewhat more extended and to exhibit a larger amplitude (e.g. $A=20$ ). However, preference can be given to the model showing superwind shells of enhanced density but preserving a $1 / r^{2}$ distribution in the various parts of the dust shell due to a better overall match of the observations.

\section{Summary and discussion}

The first diffraction-limited $2.13 \mu \mathrm{m}$ observations of NML Cyg with 73 mas resolution were presented. The speckle interferograms were obtained with the $6 \mathrm{~m}$ telescope at the Special Astrophysical Observatory, and the image reconstruction was based on the bispectrum speckle-interferometry method. In order to interpret these observations radiative transfer calculations have been carried out. We modelled the spectral energy distribution, in particular the ISO SWS spectroscopy (Justtanont et al. 1996), the $2.13 \mu \mathrm{m}$ visibility function, and mid-infrared visibility functions at $11 \mu \mathrm{m}$ (e.g. Danchi et al. 1999). Preference was given to those observations conducted in phase or close to the $2.13 \mu \mathrm{m}$ observations in June 1998.

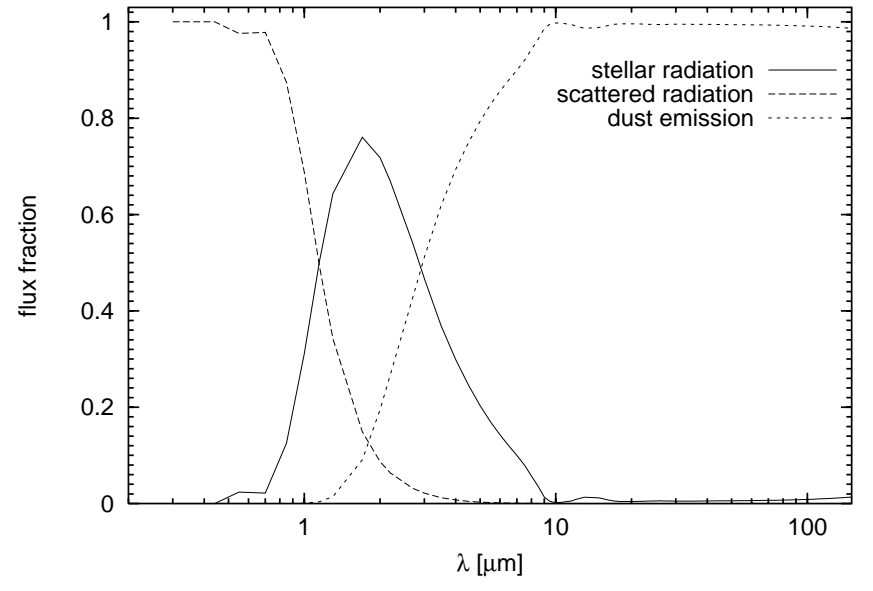

Fig. 11. Fractional contributions of the emerging stellar radiation as well as of the scattered radiation and of the dust emission to the total flux as a function of the wavelength for the model with an inner superwind shell of amplitude $A=10$ between $Y_{n 1}=2.5$ and $Y_{n 2}=3.5$ and an additional far-out density enhancement at $Y_{f 1}=30$ with $A=5$ (see Fig. 10). Model parameters are: black body, $T_{\text {eff }}=2500 \mathrm{~K}, T_{1}=1000 \mathrm{~K}$, $\tau_{0.55 \mu \mathrm{m}}=30$, Ossenkopf et al. (1992) silicates, and a Mathis et al. (1977) grain size distribution with $a_{\max }=0.15 \mu \mathrm{m}$

The observed dust shell properties do not appear to be in accordance with standard single-shell models 

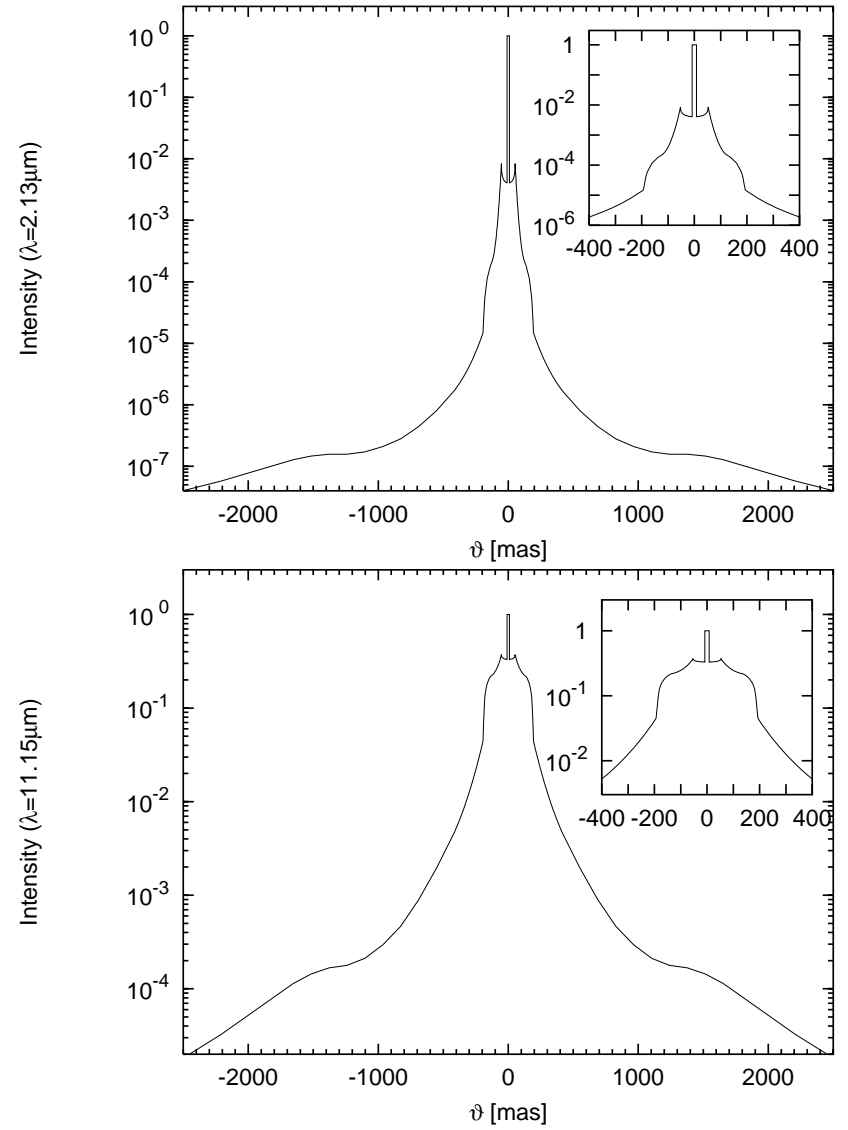

Fig. 12. Normalized intensity at $2.13 \mu \mathrm{m}$ (top) and $11.15 \mu \mathrm{m}$ (bottom) vs. angular displacement $\vartheta$ for a superwind model with an inner embedded superwind shell of amplitude $A=10$, located between $Y_{n 1}=2.5$ and $Y_{n 2}=3.5$, and an additional far-out density enhancement at $Y_{f 1}=30$ with $A=5$. The central peak belongs to the central star. The inner hot rim of the circumstellar shell has a radius of 52.5 mas, the inner embedded superwind shell extends from 131.5 mas to 184 mas, and the cool far-out component is located at a radius of 1500 mas. All loci correspond to local intensity maxima or plateaus. In the $K$ band the inner rim of the circumstellar shell is noticeably limbbrightened. The models refer to $T_{\text {eff }}=2500 \mathrm{~K}, T_{1}=1000 \mathrm{~K}$, and $\tau(0.55 \mu \mathrm{m})=30$. The calculations are based on a black body, Ossenkopf et al. (1992) silicates, and a Mathis et al. (1977) grain size distribution with $a_{\max }=0.15 \mu \mathrm{m}$

(uniform outflow) but seem to require multiple components. Since single-shell models fail to match the observations, density enhancements in the dust shell were considered. Such density enhancements correspond to previous periods of enhanced mass-loss. The episodic and/or stochastic occurence of such superwind phases seems to be a typical feature of the late stages of massive star evolution. An extensive grid of models was calculated for different locations and amplitudes of such superwind regions in the dust shell. It turned out that at least two superwind phases have to be taken into account in order to to match the observations from the optical to the sub-mm domain. The best model is that of a dust shell with a temperature of $1000 \mathrm{~K}$ at its inner radius of $6.2 R_{*}$. It includes a close embedded superwind shell extending from $15.5 R_{*}$

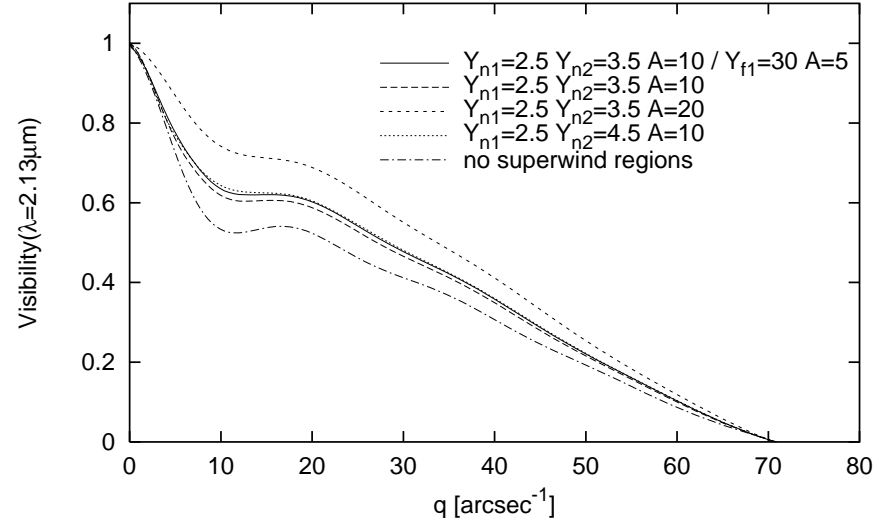

Fig. 13. Model visibilities at $2.13 \mu \mathrm{m}$ in the long-baseline interferometry range up to baselines of $36 \mathrm{~m}(6 \mathrm{~m}: q=$ $13.4 \mathrm{arcsec}^{-1}$ ) for different assumptions on the density distribution. The solid line gives the best fit to the observations and refers to a dust shell with an inner embedded superwind region with amplitude $A=10$, located between $Y_{n 1}=2.5$ and $Y_{n 2}=3.5$, and an additional far-out density enhancement at $Y_{f 1}=30$ with $A=5$. The other lines correspond to models without an outer density enhancement and a superwind shell of equal properties, of larger amplitude, and larger extension, resp. A standard model without any density enhancement is given as well. The models refer to $T_{\text {eff }}=2500 \mathrm{~K}, T_{1}=1000 \mathrm{~K}$, and $\tau(0.55 \mu \mathrm{m})=30$ and are based on a black body, Ossenkopf et al. (1992) silicates, and a Mathis et al. (1977) grain size distribution with $a_{\max }=0.15 \mu \mathrm{m}$

to $21.7 R_{*}$ with an amplitude of 10 and a far-out density enhancement at $186 R_{*}$ with an amplitude of 5 . The inner rim of the dust shell, i.e. the dust condensation zone, has a dust temperature of $T_{1}=1000 \mathrm{~K}$ and an angular diameter of 105 mas. At $2.13 \mu \mathrm{m}$ this zone is limb-brightened leading to a ring-like intensity distribution. The diameter of the embedded close superwind region extends from 263 mas to 368 mas and is composed of relatively cool dust with temperatures ranging from 583 to $430 \mathrm{~K}$. At the inner boundary of the distant superwind region the dust temperature has dropped to $149 \mathrm{~K}$ corresponding to an inner diameter of $3{ }^{\prime \prime} 15$.

Figure 13 shows the $2.13 \mu \mathrm{m}$ visibility for baselines up to $36 \mathrm{~m}$ and different assumptions on the superwind regions in comparison with a standard uniform outflow model. The central star, having a diameter of $\sim 16$ mas, becomes partially resolved for baselines larger than $10 \mathrm{~m}$ and is completely resolved at a baseline of $31 \mathrm{~m}$.

During the entire grid calculations the validity of other parameters as, optical depths, dust temperatures, grain sizes, effective temperatures, etc., was comprehensively checked. The grain sizes, $a$, were found to be in accordance with a standard distribution function, $n(a) \sim a^{-3.5}$, with $a$ ranging between $a_{\min }=0.005 \mu \mathrm{m}$ and $a_{\max }=0.15 \mu \mathrm{m}$. The effective temperature was chosen to be $2500 \mathrm{~K}$. The temperature of a giant with spectral type M6 III is $\sim 3200 \mathrm{~K}$ (Perrin et al. 1998) whereas supergiants (I-II) have systematically lower temperatures (Dyck et al. 1996, 1998). For an M6 supergiant the effective temperature can 
be estimated to be close to $3000 \mathrm{~K}$. However, (moderate) variations of the effective temperature affect the radiative transfer models only slightly. Thus, though a somewhat higher effective temperature appears to better comply with the spectral type, differences to the presented $T_{\text {eff }}=2500 \mathrm{~K}$ grid are only of minor nature and results and conclusions of the presented study remain unchanged. Corresponding test calculations have been performed for $T_{\text {eff }}=3000 \mathrm{~K}$ and $T_{\text {eff }}=3500 \mathrm{~K}$. The temperature at the inner rim of the dust shell was found to be more or less fixed at $1000 \mathrm{~K}$ in order to be in accordance with the observations. However, slightly higher temperatures, e.g. $1100 \mathrm{~K}$, appear to be possible as well. The bolometric flux amounts to $F_{\mathrm{bol}}=3.6310^{-9} \mathrm{Wm}^{-2}$ corresponding to a central-star luminosity of $L / L_{\odot}=1.1310^{5} \cdot(d / \mathrm{kpc})^{2}$. Accordingly, NML Cyg is a true massive supergiant with $L \sim 410^{5} L_{\odot}$ at the distance of the Cyg OB2 association.

Though the density distribution was scaled by amplitudes $A$ in various parts of of the dust shell, its general $1 / r^{2}$ dependence proved to be appropriate to model the observations and thus could be maintained. Alternative models were found if one considers a much steeper density distribution in the inner embedded superwind region. Then, however, the superwind shell has the same inner diameter but a larger amplitude and extension. A slight improvement of the far-infrared properties can be obtained if a shallower density distribution of $\rho \sim 1 / r^{1.7}$ is considered in the distant superwind region. Then, assuming that the outflow velocity kept constant, the massloss rate in the outer component has decreased with time. The present-day mass-loss rate can be determined to be $\dot{M}=1.210^{-4} M_{\odot} / \mathrm{yr}$. The inner embedded superwind shell corresponds to a phase of enhanced massloss $\left(\dot{M}=1.210^{-3} M_{\odot} / \mathrm{yr}\right)$ in the immediate history of NML Cyg which began $59.2 \mathrm{yr}$ ago and lasted for $\sim 18 \mathrm{yr}$ if $d=1.8 \mathrm{kpc}$ and $v=25 \mathrm{~km} \mathrm{~s}^{-1}$. Correspondingly, the outer superwind region is due to to a high mass-loss pe$\operatorname{riod}\left(\dot{M}=6.010^{-4} M_{\odot} / \mathrm{yr}\right)$ which terminated $529.3 \mathrm{yr}$ ago.

The inner diameter of the dust shell of 105 mas is close to the one found by the standard uniform-outflow models of Rowan-Robinson \& Harris (1983) and Ridgway et al. (1986) (80 mas and 90 mas, resp.) but only half as large as the one found by Monnier et al. (1997) (240 mas). Note, however, that the respective models differ in various aspects. On the one hand, the simultaneous match of SED and visibilities appear to require density distributions different from the standard uniform-outflow assumption as indicated by the models of Monnier et al. (1997) and the present models. On the other hand, Monnier et al. (1997) used a two-component model with exponentially declining density distributions that is different from the current superwind model which, in turn, shows single density jumps but preserves $1 / r^{2}$ density courses in the various dust shell parts. The present study matches the observations for a broad wavelength range, i.e. from $\sim 0.7 \mu \mathrm{m}$ to $400 \mu \mathrm{m}$. Another difference concerns the choice of other dust-shell parameters, in particular the optical constants, the dust-shell temperature and the dust-grain sizes. Furthermore, it has to be kept in mind that the observational data often belong to different epochs and in the case of long-baseline interferometry on the used position angle as well. For instance, Sudol et al. (1999) found their low spatial-frequency $11 \mu \mathrm{m}$ data not to be consistent with the higher frequency data of Monnier et al. (1997) possibly pointing to an expanding doubleshell structure (due to the different epochs) or to the existence of bipolar structures (due to the different position angles). They stress the need of coeval data covering several position angles to solve this issue. Danchi et al. (1999) presented follow-up observations of the intial measurements of Monnier et al. (1997) giving evidence of the dust-shell expansion at $11 \mu \mathrm{m}$. We note that it was essential for the current superwind models to consider the Danchi et al. (1999) data being coeval with our $K$-band measurements instead of taking only the former Monnier et al. observations. Finally, all models of NML Cyg available so far rely on the assumption of spherical symmetry whose applicablity still has to be proven. Richards et al. (1996), for instance, discussed the existence of a bipolar outflow from interferometric maser observations. Nature is certainly much more complex than the simple models given in the present study and great care has to be taken during model construction concerning its reliability and unambiguousness. Nevertheless, it can be concluded that the dust shell of NML Cyg consists of a multiple-component structure with two regions attesting the ocurrence of previous superwind phases, one in its immediate past, i.e. decades ago, and one hundreds of years ago. Thus, the red supergiant NML Cyg proves to be an important example of episodic mass-loss phases in the evolution of massive stars along the Red Supergiant Branch.

Acknowledgements. The observations were made with the SAO $6 \mathrm{~m}$ telescope, operated by the Special Astrophysical Observatory, Russia. We thank K. Justtanont for providing the ISO-SWS spectral energy distribution. The radiative-transfer calculations are based on the code DUSTY developed by Ž. Ivezić, M. Nenkova and M. Elitzur. This research has made use of the SIMBAD database, operated by CDS in Strasbourg.

\section{References}

Benson, J. M., \& Mutel, R. L. 1979, ApJ, 233, 119

Boboltz, D. A., \& Marvel, K. B. 2000, ApJ, 545, L149

Blöcker, T., Balega, Y., Hofmann, K.-H., et al. 1999, A\&A, 348,805

Bowers, P. F., Johnston, K. J., \& Spencer, J. H. 1983, ApJ, 274,733

Danchi, W. C., Monnier, J. D., Hale, D. D. S., Tuthill, P. G., \& Townes, C. H. 1999, in Optical and IR Interferometry from Ground and Space, ed. S. C. Unwin, \& R. V. Stachnick, ASP Conf. Ser., 194, 180

David, P., \& Pegourie, B. 1995, A\&A, 293, 833

Diamond, P. J., Norris, R. P., \& Booth, R. S. 1984, MNRAS, 207,611 
Dickinson, D. F., Reid, M. J., Morris, M., \& Redman, R. 1978, ApJ, 220, L113

Draine, B. T., \& Lee, H. M. 1984, ApJ, 285, 89

Dyck, H. M., \& Benson, J. M. 1992, AJ, 104, 377

Dyck, H. M., Forbes, F. F., \& Shawl, S. J. 1971, AJ, 76, 901

Dyck, H. M., Lockwood, G. W., \& Capps, R. W. 1974, ApJ, 189, 89

Dyck, H. M., van Belle, G. T., \& Thompson, R. R. 1998, AJ, 116,981

Dyck, H. M., Benson, J. A., van Belle, G. T., \& Ridgway, S. T. 1996, AJ, 111, 1705

Dyck, H. M., Zuckerman, B., Leinert, C., \& Beckwith, S. 1984, ApJ, 287, 801

Engels, D., Schmid-Burgk, J., \& Walmsley, C. M. 1986, A\&A, 167,129

Fix, J. D., \& Cobb, M. L. 1988, ApJ, 329, 290

Forbes, F. F. 1967, ApJ, 147, 1226

Gauger, A., Balega, Y., Irrgang, P., Osterbart, R., \& Weigelt, G. 1999, A\&A, 346, 505

Goss, W. M., Winnberg, A., \& Habing, H. J. 1974, A\&A, 30, 349

Gregory, P. C., \& Seaquist, E. R. 1976, ApJ, 204, 626

Habing, H. J., Goss, W. M., \& Winnberg, A. 1982, A\&A, 108, 412

Hackwell, J. A. 1974, A\&A, 21, 239

Harvey, P. M., Bechis, K. P., Wilson, W. J., \& Ball, J. A. 1974, ApJS, 27, 331

Heger, A., Jeannin, L., Langer, N., \& Baraffe, I. 1997, A\&A, 327,224

Herbig, G. H., \& Zappala, R. R. 1970, ApJ, 162, L15

Hofmann, K.-H., \& Weigelt, G. 1986, A\&A, 167, L15

Hyland, A. R., Becklin, E. E., Frogel, J. A., \& Neugebauer, G. 1972, A\&A, 16, 204

Ivezić, Ž., \& Elitzur, M. 1996, MNRAS, 279, 1019

Ivezić, Ž., \& Elitzur, M. 1997, MNRAS, 287, 799

Ivezić, Ž., Nenkova, M., \& Elitzur, M. 1997, User Manual for DUSTY, University of Kentucky

de Jager, C., \& Nieuwenhuijzen, H. 1997, MNRAS, 290, L50

Johnson, H. L. 1967, ApJ, 149, 345

Johnson, H. L. 1968, ApJ, 154, L125

Johnson, H. L., Low, F. J., \& Steimentz, D. 1965, ApJ, 142, 808

Justtanont, K., de Jong, T., Helmich, F. P., et al. 1996, A\&A, 315, L217

Knapp, G. R., Philips, T. G., Leighton, R. B., et al. 1982, ApJ, 252,616

Krüger, D., \& Sedlmayr, E. 1997, A\&A, 321, 557

Kruzewski, A. 1971, AJ, 76, 576

Labeyrie, A. 1970, A\&A, 6, 85

Langer, N., Heger, A., \& Garcia-Segura, G. 1998, Rev. Mod. Astron., 11, 57

Lee, T. A. 1970, ApJ, 162, 217

Lohmann, A. W., Weigelt, G., \& Wirnitzer, B. 1983, Appl. Opt., 22, 4028
Low, F. J., Johnson, H. L., Kleinman, D. E., Latham, A. S., \& Geisel, S. L. 1970, ApJ, 160, 531

Lucas, R., Bujarrabal, V., Guilloteau, et al. 1992, A\&A, 262, 491

McCarthy, D. W. Jr. 1979, High Angular Resolution Stellar Interferometry, IAU Coll. 50, ed. J. Davis, \& W. J. Tango, 18

Masheder, M. R. W., Booth, R. S., \& Davies, R. D. 1974, MNRAS, 166, 561

Mathis, J. S., Rumpl, W., \& Nordsieck, K. H. 1977, ApJ, 217, $425(\mathrm{MRN})$

Merrill, K. M., \& Stein, W. A. 1976, PASP, 88, 294

Monnier, J. D., Bester, M., Danchi, W. C., et al. 1997, ApJ, 481,420

Monnier, J. D., Geballe, T. R., \& Danchi, W. C. 1998, ApJ, 502,833

Morris, M., \& Jura, M. 1983, ApJ, 267, 179

Morris, M., Redman, R., Reid, M. J., \& Dickinson, D. F. 1979, ApJ, 229, 257

Neugebauer, G., Martz, D. E., \& Leighton, R. B. 1965, ApJ, 142,399

Ossenkopf, V., Henning, T., \& Mathis, J. S. 1992, A\&A, 261, 567

Perrin, G., Coudé du Foresto, V., Ridgway, S. T., et al. 1998, A\&A, 331, 619

Richards, A. M. S., Yates, J. A., \& Cohen, R. J. 1996, MNRAS, 282,665

Ridgway, S. T., Joyce, R. R., Connors, D., Pipher, J. L., \& Dainty, C. 1986, ApJ, 302, 662

Rowan-Robinson, M, \& Harris, S. 1983, MNRAS, 202, 767

Savage, B. D., \& Mathis, J. S. 1979, ARA\&A, 17, 73

Schwartz, P. R., \& Barrett, A. H. 1970, ApJ, 159, L123

Schwartz, P. R., Harvey, P. M., \& Barrett, A. H. 1974, ApJ, 187,491

Sibille, F., Chelli, A., \& Lena, P. 1979, A\&A, 79, 315

Snyder, L. E., \& Buhl, D. 1975, ApJ, 197, 329

Sopka, R. J., Hildebrand, R., Jaffe, D. T., et al. 1985, ApJ, 294,242

Strecker, D. W. 1975, AJ, 80, 451

Strecker, D. W., \& Ney, E. P. 1974, AJ, 79, 1410

Sudol, J. J., Dyck, H. M., Stencel, R. E., Klebe, D. D. D.I., \& Creech-Eakman, M.J. 1999, AJ, 117, 1609

Suh, K. W., \& Jones, T. J. 1997, ApJ, 479, 918

Vanbeveren, D., van Rensbergen, W., \& de Loore, C. 1994, Evolution of Massive Stars (Kluwer, Dordrecht)

Walmsley, C. M., Chini, R., Kreysa, E., et al. 1991, A\&A, 248, 555

Weigelt, G. 1977, Optics Commun., 21, 55

Weigelt, G. 1991, in Progress in Optics, vol. 29, ed. E. Wolf (Elsevier Science Publishers, Amsterdam), 293

Wilson, W. J., Barrett, A. H., \& Moran, J. M. 1970, ApJ, 160, 545

Winters, J. M., Fleischer, A. J., Le Bertre, T., \& Sedlmayr, E. 1997, A\&A, 326, 305 